\title{
Practical clinical trials for schizophrenia
}

\author{
SCOTT STROUP
}

\section{PRACTICAL CLINICAL TRIALS FOR SCHIZOPHRENIA}

In order to meet the requirements of regulatory agencies efficiently, efficacy trials establish the short-term safety of a drug and its ability to lessen target symptoms under ideal circumstances. These trials provide little information about how interventions will work in the real world, where clinicians exercise considerable judgment in making recommendations, patients make choices about the treatments they are offered, and where costs, convenience, and comfort have significant influence. A result is the so-called efficacy-effectiveness gap in which treatments are found less beneficial in typical circumstances rather than in the ideal circumstances of efficacy trials (Institute of Medicine, 1985).

In addition, efficacy trials do not answer many important questions faced by patients, clinicians, and policy makers. Because efficacy trials usually compare new drugs to placebo or a single active drug, they often do not provide direct information regarding a treatment's efficacy compared to the most commonly used treatments. Further, they are not concerned with many important out-

Address for correspondence: Professor S. Stroup, Department of Psychiatry, University of North Carolina School of Medicine, Chapel Hill, NC 27599-7160 (USA).

E-mail: sstroup@med.unc.edu

Declaration of Interest: Dr. Stroup receives support from NIH Research Grant \#1 K23 MH67002-01A1 funded by the National Institute of Mental Health and the Office of the Director, Office of Behavioral and Social Research (OD/OBSSR), and by the National Association of Research in Schizophrenia and Affective Disorders. The Clinical Antipsychotic Trials of Intervention Effectiveness project was supported with funds from the National Institute of Mental Health under contract NO1 MH90001. Regarding possible conflicts, in the past 2 years he has been paid as a consultant for Glaxo SmithKline and Bristol Myers Squibb and has received research support from Eli Lilly and Co. comes. For example, schizophrenia efficacy trials typically focus on reductions in psychotic symptoms as measured by a rating instrument as the primary outcome. While reduced psychotic symptoms and drug tolerability are important, other outcomes such as social functioning, work, relapse prevention, and cost effectiveness are also relevant to persons who make decisions about which treatments to offer or to use.

\section{QUALITY ISSUES IN CLINICAL TRIALS}

Bias can enter into the results and interpretation of clinical trials in numerous ways, for example through study design, selection of endpoints, trial conduct (e.g., protocol violations or early discontinuations), and definition of analysis groups (e.g., exclusion of subjects based on outcomes). Randomization and blinding of treatment are two key tools for minimizing bias. If successful, randomization and blinding minimize differential bias but they do not eliminate other biases, such as the ones mentioned above, that threaten the internal validity of studies. The Consolidated Standards for Reporting Trials (CONSORT) recommendations are intended to help make any biases apparent to readers (Moher et al., 2001; Ioannidis et al., 2004). These recommendations seek to standardize reporting so that study findings are understandable and to make evident any biases introduced through study design, conduct, analysis, or interpretation.

It is well known that funding source influences clinical trials. Recent research shows that this effect may be strongest on how the studies are reported and interpreted (Als-Nielsen et al., 2003). In particular, this research found that industry sponsorship did not diminish quality of study design or even results, but influenced the conclusions reached in publications. Because industry spon-

Epidemiologia e Psichiatria Sociale, 14, 3, 2005 
sorship is problematic, independent funding, from government agencies or foundations, is a possible solution.

Subjects in schizophrenia trials often drop out of studies or discontinue study medications before the protocolspecified time. Completion rates in schizophrenia trials seldom often approach $50 \%$ in efficacy trials, and are even lower in long-term trials. Drop-outs in schizophrenia trials represent missing data that carries information about efficacy or safety. Subjects who receive a treatment that is efficacious for them tend to remain in the study, and subjects who respond poorly tend to drop out. Similarly, subjects who have side effects or adverse events also drop out. This means that data is not missing randomly, but rather is associated with the dependent and independent variables. This type of nonrandom missing data is problematic for statistical methods for which randomness is a basic assumption. In the commonly used technique of last-observation carried forward (LOCF), the last non-missing value is used to represent later missing values. Although this technique is favored by regulatory agencies, it may cause misleading results in efficacy studies where there are many drop-outs due to side effects. Early drop-outs due to side effects, before a treatment has had time to work, can make a treatment appear less efficacious than a better tolerated comparator that has longer to work (Rosenheck, 2005).
Efficacy trials typically seek to establish short-term treatment response in comparison to placebo. However, allowing adequate time for response is needed for successful comparative studies of effectiveness. For example, even if one drug may lead to faster response, it is not clear that this predicts long-term clinical advantages if, over time, a comparator group ultimately has a similar degree of response.

\section{Practical Clinical Trials}

Practical clinical trials (practical trials) are effectiveness studies that can be thought of as hybrids of efficacy trials and large simple trials (which are discussed in this issue in an editorial by John Geddes) (see table I). The key feature of practical trials is that they have hypotheses and study designs that are formulated to provide information to help clinicians and health care policy makers make key decisions (Tunis et al., 2003). Practical trials try to answer pragmatic questions about the risks, benefits, and costs of interventions in the routine clinical situations in which they arise. Like large simple trials, practical trials recruit patients from the types of clinical settings where patients with the condition under study typically receive care, enroll a broad spectrum of patients, and compare clinically relevant interventions. The inter-

Table I. - Typical characteristics of efficacy, large simple, and practical trials.

\begin{tabular}{|c|c|c|c|}
\hline & Efficacy trials & Large simple trials & Practical trials \\
\hline Goal & To determine efficacy and safety & $\begin{array}{l}\text { To determine relative clinical } \\
\text { benefits of approved treatments }\end{array}$ & $\begin{array}{l}\text { To inform decision makers about relative } \\
\text { benefits and risks of approved treatments }\end{array}$ \\
\hline When & Before a drug is marketed & Post-market & Post-market \\
\hline Subjects & Diagnosis by structured interview & $\begin{array}{l}\text { Clinical diagnosis or structured } \\
\text { interview }\end{array}$ & Clinical diagnosis or structured interview \\
\hline Sample size & A few hundred & Thousands & Hundreds to thousands \\
\hline Comparator & $\begin{array}{l}\text { Placebo and/or a single active } \\
\text { comparator }\end{array}$ & $\begin{array}{l}\text { One or more active comparators; } \\
\text { clinical equipoise }\end{array}$ & $\begin{array}{l}\text { One or more active comparators; clinical } \\
\text { equipoise }\end{array}$ \\
\hline Dosing & Fixed or flexible dosing & $\begin{array}{l}\text { Flexible dosing in clinically } \\
\text { used range }\end{array}$ & Flexible dosing in clinically used range \\
\hline Blinding & Double-blinded & Open label & $\begin{array}{l}\text { Open label with blinded raters } \\
\text { or double-blinded }\end{array}$ \\
\hline Duration & 4-16 weeks & More than one year & 6 months or more \\
\hline Research sites & $\begin{array}{l}\text { Small number of experienced } \\
\text { research sites }\end{array}$ & $\begin{array}{l}\text { Dozens to hundreds of sites: Sites } \\
\text { are typical treatment settings }\end{array}$ & $\begin{array}{l}\text { Dozens of sites: Typical treatment settings } \\
\text { capable of implementing detailed research } \\
\text { protocol }\end{array}$ \\
\hline Research protocol & Strictly defined research protocol & $\begin{array}{l}\text { Protocol very similar to usual } \\
\text { practice }\end{array}$ & Protocol very similar to usual practice \\
\hline $\begin{array}{l}\text { Co-morbid medical and } \\
\text { psychiatric conditions }\end{array}$ & Excluded & Included & Included \\
\hline $\begin{array}{l}\text { Adjunctive and } \\
\text { concomitant treatments }\end{array}$ & Not allowed or strictly limited & Allowed as in usual practice & Allowed as in usual practice \\
\hline Outcomes of interest & $\begin{array}{l}\text { Symptom rating scales: safety: } \\
\text { other clinical parameters }\end{array}$ & $\begin{array}{l}\text { A single. well-defined. clinically } \\
\text { important outcome }\end{array}$ & $\begin{array}{l}\text { Primary outcome is clinically important } \\
\text { and discrete; multiple other outcomes, } \\
\text { including safety and costs, may be examined }\end{array}$ \\
\hline
\end{tabular}


ventions examined in practical trials are derived from the real-world choices faced by clinicians and patients. Practical trials compare treatments for which there is genuine uncertainty about relative advantages. This clinical equipoise is the justification for randomization, and makes it necessary to compare good treatments at usually effective and tolerated dosages.

By enrolling research participants who reflect the range and distribution of patients seen in clinical practice, practical trials attempt to enhance the external validity of studies in order to increase the likelihood that the results are useful to clinical and policy decision makers. Because practical trials are conducted in typical treatment settings where the ancillary care that patients receive is more typical of average care than in research settings, the generalizability of the findings may be enhanced in a way that specifically diminishes a barrier to the implementation of research findings by clinicians (Tunis et al., 2003). Further, the duration of follow-up in practical trials is longer than efficacy trials in order to better reflect the course of an illness, although for long-term illnesses like schizophrenia even trials that last more than a year might be considered "short-term".

Rather than focusing on a single definitive endpoint as in large simple trials, practical trials examine a broad range of relevant outcomes (see table I). Some outcomes are primarily of interest to clinicians, for example effects on metabolic parameters and changes in symptom and side effect rating scales. Policy makers may be particularly interested in cost-effectiveness. Practical trials also include outcomes that are oriented to the concerns of patients, for example quality of life, social and vocational functioning, and housing status. Because schizophrenia, in the relatively short follow-up periods of clinical trials, is not often associated with definitive outcomes used in other medical fields (e.g., death or myocardial infarction in cardiology trials), a variety of other outcomes have been used in schizophrenia trials that look beyond symptom reduction. These include hospitalization, relapse, remission, study drop-out, and treatment discontinuation. For example, remission is a relevant outcome for individuals with schizophrenia who enter a study with acute or persistent symptoms, functional impairment, or disability. Definitions of remission that require threshold levels of symptom resolution in addition to achievement of a threshold level of functioning are appropriate for patients seeking to resume premorbid levels of functioning. Relapse, or recurrence of symptoms or disability that reaches a predefined level of severity or requires a new intervention, is relevant for patients who have achieved remission.

\section{The CATIE Schizophrenia Trial}

The Clinical Antipsychotic Trials of Intervention Effectiveness (CATIE) schizophrenia trial, sponsored by the U.S. National Institute of Mental Health (NIMH), was designed to evaluate the effectiveness of second-generation antipsychotics and a representative first-generation antipsychotic in the treatment of chronic schizophrenia (Stroup et al., 2003). Approximately 1500 subjects with schizophrenia were recruited at more than 50 sites, including state mental health agencies, private practices, Veteran's Affairs Health Centers, and university clinics. In Phase 1, participants were randomly assigned to double-blind treatment with one of five study drugs (olanzapine, perphenazine, quetiapine, risperidone, or ziprasidone) at commonly used dosages. Patients with concurrent substance use disorders, comorbid psychiatric illnesses, and stable medical conditions were allowed to enroll (see figure 1).

The primary outcome in CATIE was all-cause treatment discontinuation as discussed below. As a practical trial, a broad range of other outcomes was also considered, including effects of the study treatments on symptoms, extrapyramidal and metabolic side effects, adverse events, substance use, violent behavior, costs and service use, neurocognitive functioning, vocational functioning, and quality of life.

The CATIE Schizophrenia Trial was designed to imitate routine clinical practice with randomizations at clinical decision points where recommendations are uncertain. Participants who discontinued the first randomly assigned medication were offered the opportunity to enter Phase 2 of the study, in which they chose one of two treatment pathways with random assignment. Participants who discontinued the first treatment because of inadequate efficacy were encouraged to enter a trial comparing clozapine to other second-generation drugs. Participants who discontinued the first treatment because of poor tolerability were encouraged to enter a trial comparing ziprasidone to other second-generation drugs. If a participant discontinued the second drug, then he or she could enter a third phase in which a selection of openlabel treatments was available based on the individual's clinical situation.

In all phases of the CATIE study flexible dosages of medicines were allowed within ranges that reflect usual care. All other medications, except for other antipsychotic drugs, were allowed unless there was a specific contraindication. The scheduled treatment period of 18 months was intended to allow examination of the longer term effects of the study drugs. 


\section{The CATIE Schizophrenia Trial}

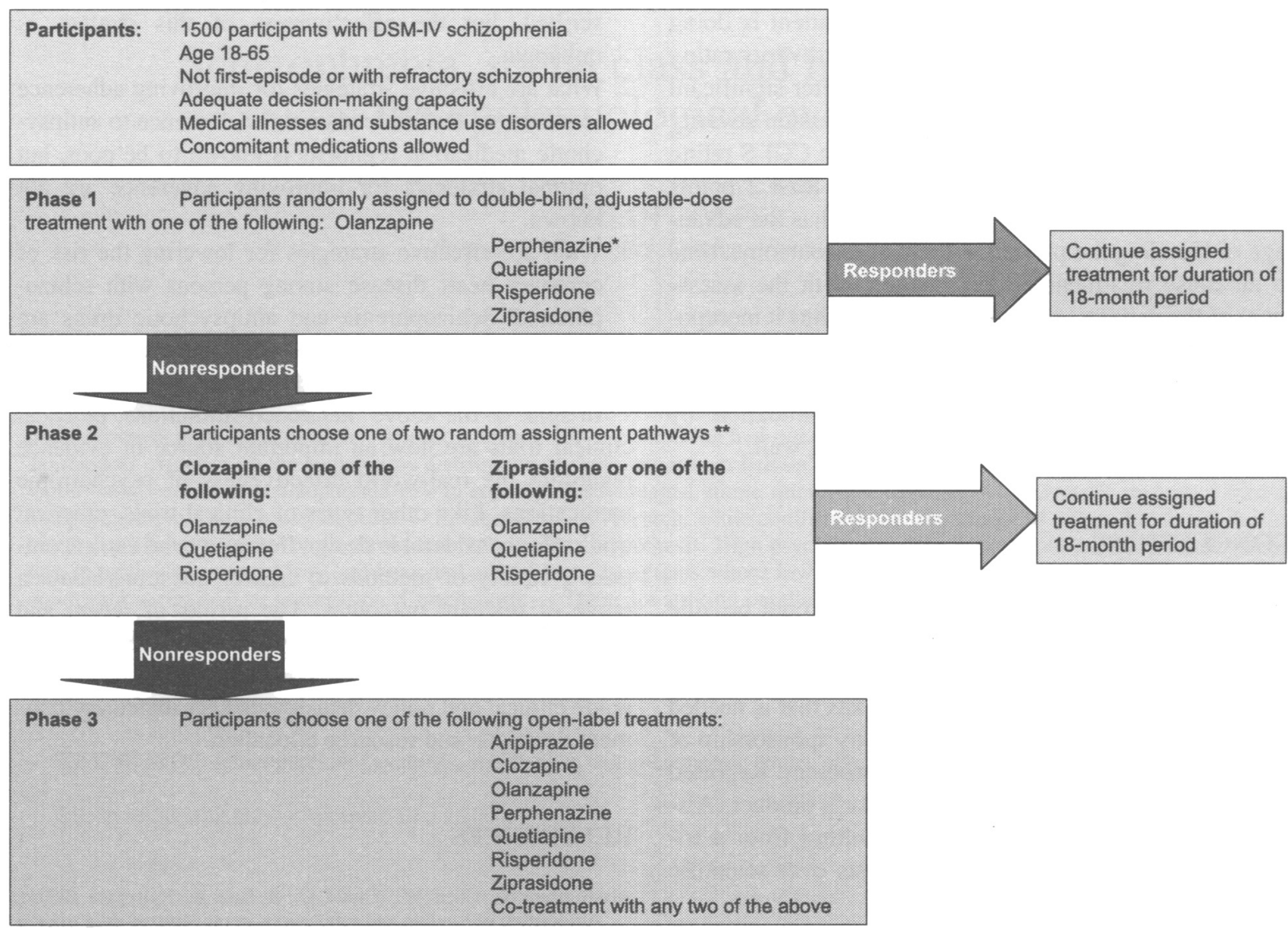

*Patients with Tardive Dyskinesia not assigned to perphenazine

"*All treatments in Phase 2 are double-blinded except for clozapine

Figure 1. - The Catie Schizophrenia Trial.

Key features of the CATIE trial as a practical trial included the following: random assignment to one of several commonly used medications on a double-blind basis; longer-term follow-up (i.e., 18 months); use of treatment discontinuation reflecting efficacy, safety and tolerability aspects of treatment as the primary endpoint; and appropriate choice of the comparator drug (i.e., use of a medium-potency conventional antipsychotic comparator and dosing of all drugs based on actual practice). In addition, a broad range of secondary endpoints relevant to decision makers were assessed.

Two outcomes in the CATIE Schizophrenia Trial are innovative. The primary outcome, time to all-cause treatment discontinuation, expected to reflect patient and clinician perceptions of efficacy, effectiveness, and tolerability, is discrete and clinically meaningful.
Although all-cause treatment discontinuation is nonspecific, it is a useful measure of a drug's overall acceptability that is important clinically because of its causes and consequences. If a patient with schizophrenia stops medications altogether, the risk of relapse and rehospitalization is greatly increased. If the cause of the discontinuation is inadequate efficacy, continued symptoms are a significant burden for the patient. Similarly, if the drug is poorly tolerated, the patient will have suffered significant discomfort, a potential medical complication, or significant morbidity. In the instances when medication changes are required or adverse events must be managed, treatment discontinuation is associated with significant increases in service use and costs as well as inconvenience for patients.

A secondary analysis in the CATIE trial will examine 
"successful treatment time" to supplement the primary all-cause treatment discontinuation outcome. This analysis will examine the amount of time a patient is doing well, defined in CATIE as having a global severity rating of mildly ill or less or as moderately ill after significant improvement (i.e., a Clinical Global Impression-severity (CGI-S) rating of mildly ill (3) or less, or a CGI-S rating of moderately ill which has improved at least 2 points from baseline). Successful treatment time has the advantage of blending the primary effectiveness outcome, time to all-cause treatment discontinuation, with the assessment of the patients' treatment response. Thus it incorporates repeated efficacy assessments without biases introduced by informative missing data, and provides a more focused assessment than time to discontinuation by counting only months that a patient is doing well.

\section{CONCLUSIONS}

Efficacy trials are internally valid but do not provide information needed by real-world clinicians on the issues they face with their patients. They do not provide information on long-term outcomes or on costs that is needed by policy makers. Further, drug company sponsorship of efficacy trials is a well-documented problem. Reported results are biased in favor of the sponsor's product (AlsNielsen et al., 2003), presumably resulting from a triumph of a company's financial interests over scientific integrity.

Practical clinical trials are one important way to evaluate the effectiveness of treatments in real-world settings. However, although the results of such randomized effectiveness trials can be invaluable to decision makers, they do not provide definitive information about whether the intervention in question will improve practice or clinical outcomes (Essock et al., 2003). Random assignment usefully removes selection biases from trials but does not mimic the way that interventions are assigned to patients in actual treatment settings. If clinicians are able to select good candidates for a new treatment, the beneficial effects may exceed what would be found in a randomized trial, where a new treatment that, for example, required extra effort from a patient would be at a disadvantage (Essock et al., 2003).

Practical clinical trials, ideally sponsored by a funding agency that is independent of the companies that make the tested interventions, are appropriate to answer current questions regarding the treatment of schizophrenia. Current topics appropriate for practical trials include the following:
1. What is the effectiveness of combination antipsychotics? Multiple medications are commonly prescribed, but the effectiveness of this strategy is unknown.

2. What are effective strategies for improving adherence to treatment recommendations? Adherence to antipsychotic medication regimens is known to be poor, but optimal strategies for improving adherence are not known.

3. What are effective strategies for lowering the risk of coronary heart disease among persons with schizophrenia? Schizophrenia and antipsychotic drugs are both associated with substantial metabolic adverse effects that are risk factors for coronary heart disease.

In spite of the above mentioned limitations, practical clinical trials are now an important source of evidence regarding the real-world effectiveness of psychotropic medications. Like other types of clinical trials, practical trials offer considerable design flexibility and can encompass a variety of methods to answer questions about a range of relevant outcomes. The results of recent and ongoing practical trials, such as the CATIE Schizophrenia Trial, should yield crucial information to guide clinical and policy decision makers regarding treatment decisions and resource allocation.

\section{REFERENCES}

Als-Nielsen B., Chen W., Gluud C. \& Lise L. Kjaergard (2003). Association of funding and conclusions in randomized drug trials: a reflection of treatment effect or adverse events? Journal of American Medical Association 290, 921-928.

Essock S.M., Drake R.E., Frank R.G. \& McGuire T.G. (2003). Randomized controlled trials in evidence-based mental health care: getting the right answer to the right question Schizophrenia Bulletin $29.115-123$.

Ioannidis J.P., Evans S.J., Gotzsche P.C., O'Neill R., Altman D.G., Schulz K. \& Moher D. for the CONSORT Group (2004). Better reporting of harms in randomized trials: an extension of the CONSORT statement Annals of Internetional Medicine 141, 781-788.

Institute of Medicine (1985). Assessing Medical Teclnologies. National Academy Press: Washington, DC.

Moher D., Schulz K.F. \& Altman D. (2001). The CONSORT statement: revised recommendations for improving the quality of reports of parallel-group randomized trials. Journal of American Medical Association 285, 1987-1991.

Rosenheck R.A. (2005). Open forum: effectiveness versus efficacy of second-generation antipsychotics: haloperidol without anticholinergics as a comparator Psychiatric Senvices 56, 85-92.

Stroup T.S., McEvoy J.P., Swartz M.S., Byerly M.J., Glick I.D., Canive J.M., McGee M.F., Simpson G.M., Stevens M.C. \& Lieberman J.A. (2003). The NIMH-CATIE Project: Schizophrenia Trial Design and Protocol Development. Schizophrenia Bulletin 29(1), 15-31.

Tunis S.R., Stryer D.B. \& Clancy C.M. (2003). Practical clinical trials: increasing the value of clinical research for decision making in clinical and health policy. Joumal of American Medical Association $290,1624-1632$ 